\title{
Buchempfehlung
}

\author{
Alexandra RamssI-Sauer
}

(C) The Author(s) 2019

\section{René Reichel}

„Vom Sinn des Sterbens. Gedanken und Anregungen für den Umgang mit Sterben und mit Sterbenwollen“"

facultas Universitätsverlag, Wien, 254 Seiten, ISBN 978-3-7089-1543-2, Euro 24,90.

Der erste Teil des Buchs handelt vom Sterben selbst und spannt den Bogen vom Sterbeprozess über die Trauer bis hin zur Auseinandersetzung mit der eigenen Endlichkeit. Praktische Gedanken und Anregungen für Hinterbliebene sowie professionelle Helfer_innen werden ebenso geteilt. Und die Fragen gestellt wie Sterbende diesen Prozess durchleben und wie es jenen geht, die Sterbende begleiten. Zudem werden Besonderheiten im Zusammenhang mit dem Thema Sterben und Kinder herausgearbeitet. Der zweite Teil des Buchs beleuchtet unterschiedlichste Perspektiven des "Sterbenwollens", Suizid und Suizidversuche und bietet Anregungen für den Umgang mit suizidalen Menschen, aber auch für Betroffene. Wobei Reichel dabei auch auf das in diesem Zusammenhang durchaus kritisch zu hinterfragende Wort „wollen“ eingeht.

Alexandra Ramssl-Sauer ( $\square)$

Palffygasse 2/1, 2500 Baden,

Österreich

kontakt@gestalttherapie-praxis.com
Es fließen laufend Konzepte der Integrativen Therapie ein. Nicht eingegangen wird auf das Sterben im Krieg. Dafür muss man auf weitere Literatursuche gehen. Die Rezension konzentriert sich etwas mehr auf den zweiten Teil des Buchs - passend zum Schwerpunktheft dieser Ausgabe von psychotherapie forum „Depression und Suizidalität“.

René Reichels Buch thematisiert das Sterben. Und das - wie er es nennt und kritisch hinterfragt - Sterbenwollen. Es werden gesellschaftliche, philosophische, rechtliche sowie wissenschaftliche Aspekte des Sterbens bzw. rund um den Sterbeprozess diskutiert. Bereits in der Einleitung hebt Reichel hervor, dass Schreiben über Tod und Sterben nie ganz objektiv sein kann, da diese existenziellen Themen unmittelbar mit Lebenserfahrungen verbunden sind. Über die künstlerische Perspektive gelingt ein flüssiger Einstieg in ein komplexes Thema. Von allgemeinen Aspekten des Sterbens leitet Reichel hin $\mathrm{zu}$ persönlichen Erfahrungen und Möglichkeiten des Denkens, Fühlens und Handelns hinsichtlich vieler Fragen für Hinterbliebene sowie professionelle Helfer_innen. Reichel schreibt „Hinterbleibende", denn seines Erachtens nach spiegelt dieser Begriff den Prozess, den man nach dem Tod eines nahestehenden Menschen durchmacht, besser wider.

Reichel geht auf den gesellschaftlichen Wandel hinsichtlich Suizid ein und beleuchtet die Wandlung eines früher eher philosophischen, theologischen oder soziologischen Themas in Richtung krankheitswertige Störung. Er wagt einen gesellschaftlichen Blick auf das Phänomen der Selbsttötung. Und stellt die Frage: „Seit wann ist Suizidalität eine Krankheit?". Reichel schildert die Historie von Suizid als Sünde über Straftat, Ehre, Verzweiflung über gesellschaftliche Zustände bis hin zu Erwin Ringel und der Sichtweise, dass Suizid eine krankheitswertige Störung ist. Er arbeitet die Feinheiten der Sprache und Begrifflichkeiten in Bezug auf Suizid heraus. Sich auf Referenzen beziehend kommt der Begriff Selbsttötung als Alternative zum Fachbegriff Suizid ins Spiel. Kritisch werden unter anderem auch weitere Begrifflichkeiten wie „Bilanzselbstmord“ oder „erweiterter Selbstmord“ hinterfragt. Widersprüche und Komplexität des Themas Suizidalität werden aufgegriffen. Reichel beleuchtet das Spannungsfeld, in das Ärzt_innen und Therapeut_innen in der Arbeit mit suizidalen Patient_innen kommen können - unter Betrachtung der rechtlichen Rahmenbedingungen. Auch Genderaspekte sowie künstlerische Einflüsse werden behandelt.

Reichel resümiert unter anderem, dass die Komplexität der Suizidalität Vielschichtigkeit im Umgang mit dieser erfordert. Er illustriert ein Kontinuum zwischen Leben und Sterben wollen angelehnt an das Salutogenese-Modell von Aaron Antonovsky, der unter ande- 
rem das Kontinuum zwischen gesund und krank sein beschrieb. Reichel bietet in diesem Abschnitt auch Anregungen zur Selbstreflexion. In der Folge beschreibt er Psychodynamiken in Bezug auf Suizid bzw. Suizidgedanken.

Das Buch bietet im letzten Abschnitt praktische Anregungen für Betroffene sowie professionelle Helfer_innen bei Suizidalität und Suizid(versuch). Unter anderem einen Fragebogen zur Selbstreflexion, Präventionsstrategien, Hilfestellungen hinsichtlich Kommunikation sowie Hilfen für Angehörige. Er spart nicht aus, dass Suizid auch in professionellen Einrichtungen vorkommen kann und ergänzt dies durch Erfahrungsberichte.

Das letzte Kapitel des Buches thematisiert Zuversicht und Verbundenheit, Lebenswillen und Lebenskraft bis hin zum Leben mit den Gegensätzen: Leben und Sterben.

Das Buch bemüht sich um eine gute Verständlichkeit, was auch gelungen ist. Es ist leicht und flüssig zu lesen und kann Widersprüche nebeneinander stehen lassen. Über das Inhalts- und Stichwortverzeichnis kann man sich einfach orientieren und auch nur punktuell in das Buch einsteigen.

Open Access. Dieser Artikel wird unter der Creative Commons Namensnennung 4.0 International Lizenz (http:// creativecommons.org/licenses/by/4.0/ deed.de) veröffentlicht, welche die Nutzung, Vervielfältigung, Bearbeitung, Verbreitung und Wiedergabe in jeglichem Medium und Format erlaubt, sofern Sie den/die ursprünglichen Autor(en) und die Quelle ordnungsgemäß nennen, einen Link zur Creative Commons Lizenz beifügen und angeben, ob Änderungen vorgenommen wurden. 